\title{
Multiple-choice questions
}

\section{Multiple-choice questions in gastro-enterology and hepatology}

\author{
M C Bateson, R Holden
}

Are the statements listed beneath each question true or false? Answers are given overleaf.

\section{QUESTION 1}

Which cranial nerves are involved in normal functioning of the gastrointestinal tract:
A II
B IV
C VI
D VIII
E X

\section{QUESTION 2}

Liver abnormalities definitely associated with Crohn's disease include:
A Steatosis
B Pericholangitis
C Amyloid
D Primary sclerosing cholangitis
E Cirrhosis

\section{QUESTION 3}

In childhood gastro-oesophageal reflux disease:

A Respiratory symptoms are common

B Barium swallow is the best test

C Columnar lined epithelium is rare

D Thickening infant feed and avoiding overfeeding are important

E Metoclopramide is useful treatment

\section{QUESTION 4}

False-negative direct urease tests for $\mathrm{Heli}$ cobacter pylori are:

A Rare

B Caused by gastrointestinal haemorrhage

Bishop Auckland General Hospital, Bishop Aukland, County Durham DL14 6AD, UK

M C Bateson

Monklands Hospital, Airdrie, UK

R Holden

Accepted 16 December 1997
C Related to recent treatment with antibiotics, bismuth and proton pump inhibitors

D Caused by ranitidine

E Identifiable by ELISA serology

\section{QUESTION 5}

Drugs with prominent hepatic and gastrointestinal effects in overdose are:

A Co-phenotrope

B Co-trimoxazole

C Co-proxamol
D Co-codamol
E Co-amilofruse

\section{QUESTION 6}

Drug overdosage in parkinsonian patients may cause pronounced gastrointestinal side-effects related to::
A Bromocriptine
B Co-beneldopa
C Benzhexol
D Selegeline
E Amantadine

\section{QUESTION 7}

What cranial nerves are involved in normal functioning of the gastrointestinal tract:

A I

B III

C V

D VII

E IX

\section{QUESTION 8}

The following hormones are important to the normal function of the gut:

A Gastrin

B Secretin

C Hydrogen potassium ATPase

D Cholecystokinin-pancreozymin (CCK-PZ)

E Vaso-active intestinal peptide (VIP)

\section{QUESTION 9}

In carcinoma of the colon:

A Most cancers arise in adenomatous polyps

B Familial polyposis coli is inherited by a recessive gene on chromosome 5

C Progression from polyp to cancer takes on average four years

D High animal fat and high vegetable intake are risk factors

E All patients with ulcerative colitis have an increased risk of carcinoma of the colon 
Answers

\section{QUESTION 1}

A False

B False

C False

D False

E True

The vagus nerve is extensively important for swallowing, gastric acid secretion and motility.

\section{QUESTION 2}

A True

B True

C True

D True

E False

Any link between cirrhosis and Crohn's disease is dubious, though there may be an increase because of transfusion-related viral hepatitis or end-stage primary sclerosing cholangitis. It is important to look for other aetiologies such as alcohol if cirrhosis does occur in these patients.

\section{QUESTION 3}

A True

B False

C True

D True

E False

Up to $10 \%$ of childhood gastro-oesophageal reflux disease patients have associated respiratory symptoms, possibly linked to aspiration. The best objective test is $24-\mathrm{h}$ oesophageal $\mathrm{pH}$ monitoring. Metoclopramide is contraindicated for those under 20 years because of severe dystonic reactions. Cisapride is probably the best prokinetic drug for children.

\section{QUESTION 4}
A False
B True
C True
D False
E True

After proton pump inhibitor and antibiotic therapy it is best to wait a month before repeat endoscopy for gastric biopsy and urease testing. The same is true of the urea breath test which is a convenient noninvasive alternative. A urease test at the time of haematemesis will usually be negative. Even after definite eradication of Helicobacter pylori, serology will remain positive for months. ELISA testing would confirm a true positive urease test.

\section{QUESTION 5}
A True
B False
C True
D True
E False

Both co-codamol and co-proxamol contain paracetamol which may be hepatotoxic. Cophenotrope has the constipating opioid diphenoxylate, but includes atropine whose unpleas- ant anticholinergic side-effects, including dry mouth, are designed to discourage over-use.

\section{QUESTION 6}
A True
B False
C True
D False
E False

Bromocriptine's usefulness is severely limited by nausea and vomiting, and this is a problem even in therapeutic doses. Benzhexol and other atropinics may cause dry mouth, gastrooesophageal reflux and constipation. However, the most serious adverse effects for these drugs are neuropsychiatric.

\section{QUESTION 7}

\section{A True \\ B False \\ C True \\ D True \\ E True}

Taste sensation is largely derived from smell, so the first nerve is involved as well as the seventh and ninth nerves which innervate the tongue. The fifth nerve serves the muscles of mastication. The ninth nerve is also important in swallowing.

\section{QUESTION 8}

A True

B True

C False

D True

\section{E False}

Gastrin stimulates gastric acid secretion and secretin stimulates bicarbonate-rich pancreatic secretion. CCK-PZ both stimulates gallbladder contraction and pancreatic enzyme secretion. Hydrogen potassium ATPase is an enzyme which determines the activity of the gastric proton pump. Though VIP excess is known to cause Verner-Morrison syndrome (watery diarrhoea, hypokalaemia, and achlorhydria) its role in health is doubtful.

\section{QUESTION 9}

\section{A True \\ B True \\ C False \\ D False \\ E False}

The adenoma-dysplasia-carcinoma sequence probably occurs in all colon cancers; however, stalked polyps are less common in the right side of the colon and cancers can arise in flat, often small, adenomas. The interval for transition from adenoma to carcinoma is on average 10 years. High fat and low fibre intake is positively associated with increased risk of colon cancer. Increased intake of fruit and vegetable is probably protective. Only patients who have ulcerative colitis involving most of the colon have significantly increased risk, which begins to rise 8 years after diagnosis. 